\title{
Semiflexible polymer condensates in poor solvents: Toroid versus spherical geometries
}

\author{
I. C. B. Miller, ${ }^{1}$ M. Keentok, ${ }^{2}$ G. G. Pereira, ${ }^{2,3}$ and D. R. M. Williams ${ }^{1}$ \\ ${ }^{1}$ Research School of Physical Sciences and Engineering, Australian National University, Canberra 0200, Australia \\ ${ }^{2}$ Department of Mechanical Engineering, University of Sydney, Sydney, 2006, Australia \\ ${ }^{3}$ MacDiarmid Institute for Advanced Materials and Nanotechnology, School of Chemical and Physical Sciences, \\ Victoria University of Wellington, P.O. Box 600, Wellington, New Zealand
}

(Received 21 July 2004; published 14 March 2005)

\begin{abstract}
Semiflexible polymers, such as DNA, in the presence of a condensing agent often form toroids. This is due to a balance between bending and surface area free energy penalties. Here we show why in experiments all the toroids have been found to have similar physical size. We also introduce a novel morphology, that of the hollow sphere, which is favored for long polymer chains. This offers the possibility of encapsulating material inside a "vesicle" made of semiflexible polymers. We also consider the case of many such polymer chains placed in a poor solvent. We show a transition between two morphologies occur on increasing concentration of polymer chains, from a thickened toroid to a spherical globule.
\end{abstract}

DOI: 10.1103/PhysRevE.71.031802

PACS number(s): 61.41.+e, 61.20.Gy, 61.25.Hq

\section{INTRODUCTION}

Perhaps the most important and fundamental problem in polymer science is the conformation of a polymer chain under different solvent conditions. For "fully flexible" chains, which suffer negligible free energy penalty upon bending, the solution to this problem has long been known [1]. In good solvents the chain is a swollen random coil, whereas in poor solvents where the monomer-monomer interactions are attractive the chain forms a fairly uniform spherical globule. This kind of behavior is seen in wide range of common synthetic polymers. However, many polymers are in fact "semiflexible;" i.e., they incur a substantial free energy penalty when bent. These include several synthetic polymers and almost all biopolymers, such as DNA and actin. The conformations of these chains are much less well understood than their more flexible cousins. Indeed, one of the most remarkable properties of DNA is the formation of toroids under poor solvent conditions [2-18]. This can be achieved by using a single solvent or by using a "condensing agent," which acts as a glue between the chain segments.

The usual argument for why toroids form is that in a toroid the chain suffers less bending energy than in a sphere, where presumably very tight bends must take place. The size of the toroid is then given as a balance between the bending energy, which favors a weak bend and hence large toroids, and the surface energy, which favors a toroid as compact as possible [15]. At least two major problems remain for single stiff chains. The first is that the size of all the toroids found experimentally is almost the same and varies very weakly with the length of chain involved. The second is that it is not clear that a toroid is the lowest-energy configuration. In this paper we show that these two problems are related. In fact we show that for long enough chains a toroid is not the best configuration and that a hollow sphere is of lower free energy. This fact means that the range of chain length over which toroids are stable is fairly small. For small chain lengths a "straight line" is the best configuration, while for longer lengths the hollow sphere is preferable. This fact, combined with the very weak $L^{1 / 5}$ dependence of the toroidal radius on chain length explains why toroids all have approximately the same size. The new "hollow sphere" morphology is of course of interest in itself. It suggests that vesicles could be made of semiflexible chains. Vesicles are already important in problems of drug delivery, and this novel form of vesicle might have interesting practical properties-for instance, the wall thickness can be readily controlled by changing the length of the polymer.

Another problem of interest is the conformation of many stiff polymer chains under the effect of a condensing agent or in a poor solvent. Given that one polymer chain will form a toroidal morphology the obvious question to ask what morphology will many chains form? Will it still be a toroid, albeit thickened up, or can another morphology be stable. On surface energy grounds, it is clear a spherical geometry would be a better geometry. We therefore develop a model of a spherical globule (without any holes in the center) and compare its free energy with that of a toroid. We show there exists a crossover (in number of chains) from a toroidal morphology to a spherical globule.

We consider a semiflexible chain of length $L$ with bending constant $\epsilon$, such that when the chain is bent with radius of curvature $R$ a bending penalty of $1 / 2 \epsilon L R^{-2}$ is incurred. We assume, as a concrete example, that the chain cross section is a square of side $a$, so that the total volume of the chain is $V=L a^{2}$. The chain is immersed in a poor solvent and the chain-solvent surface tension is $\gamma$ so that the free energy of the chain in a rodlike configuration is $4 a L \gamma$. It is useful sometimes to describe the chain in terms of two alternative parameters: the persistence length $P=\epsilon / k T$ and a dimensionless measure of the surface tension $g$, such that $\gamma=g k T / a^{2}$. In general we expect $g$ will be somewhat larger than unity.

The rest of the paper is structured as follows: In Sec. II we consider the morphologies formed by single chains as a function of increasing chain length (or conversely decreasing persistence length). Section III then involves many chained systems, and we consider and compare the free energies of the thickened toroid and a dense sphere. Finally in Sec. IV we summarize our results and give some future applications of our work. 


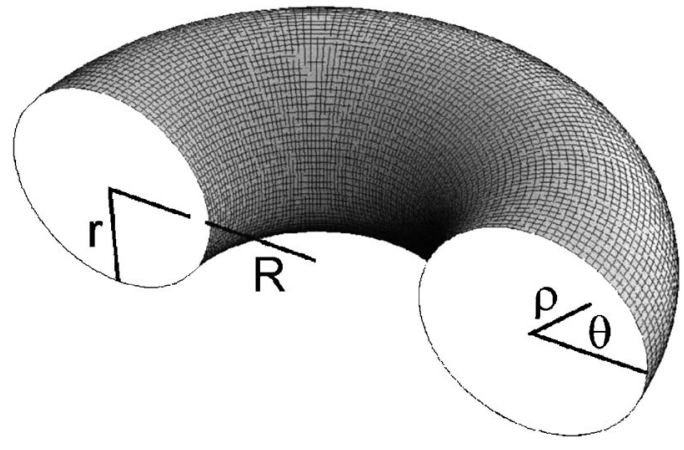

FIG. 1. A diagram of the parameters used in the toroidal calculations. $R$ is the major radius, $r$ is the minor radius, and $\rho, \theta$ are the variables for integration over the cross section.

\section{SINGLE-CHAIN SYSTEMS}

For short chains a rod will be the favored configuration. However, for longer chains it is clear that the chain can reduce its energy by looping in on itself to form a circle of radius $R$ [16]. This reduces the surface energy, since two sides of the chain can be adjacent to each other rather than solvent, but at the cost of some bending energy. The free energy difference between this prototoroid and the unbent rod is

$$
\Delta F=\frac{1}{2} \epsilon L R^{-2}-2 a \gamma(L-2 \pi R) .
$$

This is minimized for $R^{3}=V^{4 / 3} /(4 \pi a \alpha)$. Here we have used the characteristic parameter $\alpha \equiv a^{2} V^{1 / 3} \gamma / \epsilon$ first introduced by Ubbink and Odijk [15]. It turns out that $\alpha$ controls the phase behavior of the system. The critical value of $\alpha$ where the system jumps from a rod to a prototoroid is when $\Delta F=0$ or

$$
\alpha_{t c}=\frac{27}{4} \pi^{2} \frac{a^{5}}{V^{5 / 3}} .
$$

This gives us where the rod becomes unstable to toroid formation. Most toroids consist of many turns, and we need to calculate the energy of this system. We adopt the assumption of Ubbink and Odijk: that the toroid is best described as a constant density melt. This should be contrasted with the opposite limit of a "low-density" toroid, where a virial expansion in the polymer density is appropriate $[12,17]$. The simplest model is that of a toroid with major radius $R$ and with circular cross section with minor radius $r$. The circular cross section is only an approximation, but one which is sufficient for a first calculation. Using two theorems of Pappus, the surface energy and volume of this system are $F_{\text {sur } f}=4 \pi^{2} \gamma R r$ and $V=2 \pi^{2} R r^{2}$. To calculate the bending energy we use a polar coordinate system, $(\rho, \theta)$ located at the centroid of the cross section (Fig. 1). A chain passing through this point is at a distance $R+\rho \cos \theta$ from the axis of the toroid. The volume of chain located between in an element $d \rho d \theta$ is $d V=2 \pi(R+\rho \cos \theta) \rho d \rho d \theta$. The total length of chain crossing this element is $d L=d V / a^{2}$, and the energy of this segment is $d F=1 / 2 \epsilon(R+\rho \cos \theta)^{-2} d L$. Integrating this in the region $0<\theta<2 \pi$ and $0<\rho<r$, we find a bending en- ergy $2 \pi^{2} \epsilon a^{-2}\left[R-\left(R^{2}-r^{2}\right)^{1 / 2}\right]$ for the whole toroid. Eliminating the minor radius by the volume constraint and introducing a dimensionless radius $\mathcal{R} \equiv R / V^{1 / 3}$, we can write the dimensionless free energy as $\mathcal{F}_{\text {tor }}=F a^{2} \epsilon^{-1} V^{-1 / 3}$, or

$$
2 \pi^{2}\left(\mathcal{R}-\sqrt{\mathcal{R}^{2}-\frac{1}{2 \pi^{2}} \mathcal{R}^{-1}}\right)+2 \sqrt{2} \pi \alpha \sqrt{\mathcal{R}}
$$

We can then minimize this over the major radius to obtain the free energy as a function only of $\alpha, \mathcal{F}(\alpha)$. To lowest order in $\alpha$ we find the minimum lies at $\mathcal{R} \approx\left(2 \pi^{2} \alpha^{2}\right)^{-1 / 5}$ and the free energy is

$$
\mathcal{F}=\frac{5}{2}\left(2 \pi^{2}\right)^{2 / 5} \alpha^{4 / 5}+\frac{1}{8} \alpha^{2}, \quad \alpha \ll 1 .
$$

Provided $\alpha<1$ we can drop the $\alpha^{2}$ term to an accuracy of about $1 \%$.

This free energy is useful because it can be compared with that of other morphologies. It is natural to ask what other morphologies are likely. As more and more chain is placed in a toroid the toroid becomes "fatter"- the minor radius approaches the major radius. Furthermore, the major radius scales as $L^{1 / 3}$ as it would for a sphere. Essentially the toroid is attempting to become a sphere, but cannot since we have imposed a toroidal morphology. We previously rejected a spherical globule because of the presence of tight bends. However, these can be avoided by adopting the model of a hollow sphere, with inner radius $R_{0}$ and outer radius $R_{1}$. We assume the chain wraps around the center of the sphere. The free energy in this case is simple to calculate. Between radii $r$ and $r+d r$ there is a volume of chain $4 \pi r^{2} d r$ and hence a length of chain $d L=4 \pi r^{2} d r / a^{2}$. The bending energy of this portion is then $1 / 2 d L / r^{2}=2 \pi \epsilon a^{-2} d r$. The total bending energy is then the integral of this:

$$
F_{\text {bend }}=2 \pi \epsilon a^{-2}\left(R_{l}-R_{0}\right) .
$$

What is important and perhaps surprising about this result is that as $R_{0} \rightarrow 0$ the free energy approaches a finite value. Thus, even though near the center of a sphere the bend is very tight, the portion of chain undergoing that bend is very small; hence, the free energy becomes finite due to a cancellation of two terms in $r^{2}$. The surface energy is $F_{\text {sur } f}$ $=4 \pi \gamma\left(R_{1}^{2}+R_{0}^{2}\right)$. There is again a volume constraint which relates the inner and outer radii, $V=\frac{4}{3} \pi\left(R_{1}^{3}-R_{0}^{3}\right)$. The dimensionless free energy is then

$$
\begin{aligned}
\mathcal{F}_{\text {sphere }}= & 2 \pi\left[\beta^{-1 / 3}\left(1+\beta r_{0}^{3}\right)^{1 / 3}-r_{0}\right] \\
& +4 \pi \alpha\left[\beta^{-2 / 3}\left(1+\beta r_{0}^{3}\right)^{2 / 3}+r_{0}^{2}\right],
\end{aligned}
$$

where $\beta \equiv 4 \pi / 3$ and $r_{0}$ is a dimensionless radius, $r_{0}$ $\equiv R_{0} / V^{1 / 3}$. For small $\alpha \ll 1$ we find

$$
r_{0} \approx \frac{1}{2 \pi^{1 / 4} \alpha^{1 / 4}}\left(1-\frac{3}{4} \frac{\alpha^{3 / 4}}{\pi^{1 / 4}}\right)
$$

and the free energy is

$$
F \approx 4 \sqrt{\pi} \sqrt{\alpha}+\frac{11}{6} \alpha^{2} .
$$




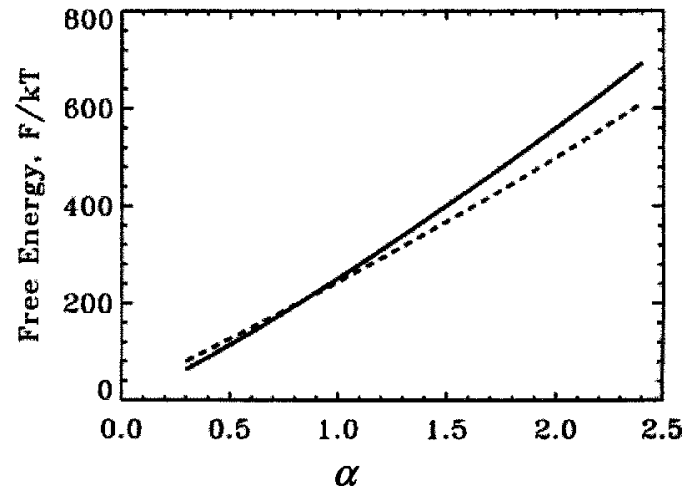

FIG. 2. The free energy vs $\alpha$ for the toroid and hollow sphere. The solid line is the free energy of the toroid and the dashed that of the hollow sphere. The critical value of $\alpha \approx 0.8423$ occurs where the two free energies intersect.

We can now determine if this free energy is lower than that of the toroidal morphology and where this occurs. In fact, for large enough $\alpha$ (long enough chains), the hollow sphere has lower free energy than the toroid. Using only the lowest-order expansion in $\alpha$ in both cases gives a critical value of $\alpha_{c}=\frac{256}{625} 10^{2 / 3} / \pi \approx 0.6$. A more accurate value (see Fig. 2 which plots the free energy of both morphologies versus $\alpha$ ) can be obtained numerically, this gives $\alpha_{c} \approx 0.8423$. It is interesting to know what the toroid and sphere look like near the transition point (Fig. 3). At this point we find a ratio of the minor radius to the major radius of the toroid of 0.455 . The toroid is thus still fairly thin. At the transition the dimensionless inner radius of the sphere is $r_{0} \approx 0.201$ and the ratio of the outer to the inner radius is $r_{1} / r_{0}=3.04$. The ratio of the inner volume to the total volume is about $3 \%$. This means that the sphere has thick walls with a small inner chamber. As more polymer is added the walls get thicker and the inner radius shrinks. Very good approximations (accurate to less

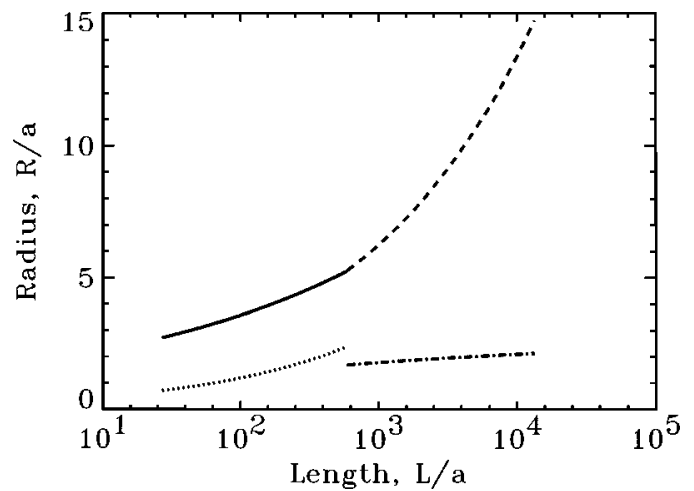

FIG. 3. The radii verses length for the toroid and hollow sphere. The solid line and the dotted lines are the major and minor radii of the toroid. The dashed and dot-dashed lines are the outer and inner radii of the hollow sphere. $P / a=30$ and $g=3$ for this graph but these values only affect the scaling of the graph and not the actual shape. The minimum length value corresponds to the change from a straight rod to a prototorus. Note the major radius of the toroid and the outer radius of the hollow sphere appear to be nearly equal at the phase boundary. This is a universal result, but appears to be a coincidence. than $1 \%$ for $\left.\alpha>\alpha_{c}\right)$ are $r_{1} / r_{0}=1+(48 / \pi)^{1 / 3} \alpha$ and $r_{0}$ $=[3 /(4 \pi)]^{1 / 3}\left\{\left[1+(48 / \pi)^{1 / 3} \alpha\right]^{3}-1\right\}^{-1 / 3}$. One interesting point concerns the behavior of the inner radius as $\alpha$ is made larger, which corresponds to increasing the chain length. We find $r_{0} \rightarrow 1 / 4 \alpha^{-1}$, which in dimensional units is $R_{0}$ $\rightarrow 1 / 4 \epsilon a^{-2} \gamma^{-1}=1 / 4(P / g)$. The hole radius will thus typically be $50 \AA$-i.e., somewhat bigger than a molecular size. This result is independent of the chain length and means that for long chains the inner radius shrinks to a constant limit, but never shrinks to zero. Thus, as the chain gets longer the inner hole has a roughly constant volume and the outer shell grows. It is perhaps interesting to compare the hollow sphere morphology with the globules found in flexible chain systems. In the latter, the inner region is actually the most dense region of the globule, whereas in our morphology exactly the opposite is true. We are now in a position to discuss in detail some of the reasons why all toroids are roughly the same size. The first of these is the formula for the toroidal radius. In dimensional units, provided $\alpha$ is less than unity this is $R$ $\approx\left(a^{2} P^{2} L / 2 \pi^{2} g^{2}\right)^{1 / 5}$. From this formula we see one simple and clear reason why all toroids are of similar size- the dependence of the toroidal radius on the parameters is very weak. In particular, it depends only on the $1 / 5$ power of the chain length. Moreover, it depends only very weakly on the surface tension and persistence length, which in any case cannot vary very much. The second reason for same-size behavior should also be fairly clear. One might think that by putting enough material into a toroid-i.e., by increasing $L$ sufficiently - the toroid could be made large. However, increasing $L$ means increasing $\alpha$, and we have shown that beyond a very low value of $\alpha \approx 0.8$ the hollow sphere is the preferred configuration. We can in fact be more quantitative than this. Suppose we take a series of chains with a given persistence length $P$ and surface tension $\gamma=g k T / a^{2}$. We then vary the length of the chain. Let us call the radius of the the smallest prototoroid that can form $R_{\text {small }}$, and the radius of the largest toroid that can form before a sphere takes over, $R_{\text {big. }}$. It is then straightforward to show that

$$
\frac{R_{\text {small }}}{R_{\text {big }}}=\frac{\sqrt{3}}{2} \frac{\left(2 \pi^{2}\right)^{1 / 5}}{\alpha_{c}^{3 / 5}}\left(\frac{P}{g a}\right)^{-1 / 2}=1.74\left(\frac{P}{g a}\right)^{-1 / 2} .
$$

Typical values of the persistence length and surface tension are $P / a=30$ and $g=3$. This gives $R_{\text {small }} / R_{\text {big }} \approx 0.55$. The dependence of this answer on $P$ and $\gamma$ is weak, so that a value of 0.5 will be typical. This implies that the range of toroidal radii will typically be of order 2 , thus showing explicitly how the small range found experimentally might be obtained. Another quantity of interest experimentally is the ratio of the minor and major radii of the toroids. This increases as the chain length, or $\alpha$, increases. It reaches a maximum value of 0.455 at $\alpha=\alpha_{c}$. Experimentally, there seem to be no toroids with a ratio greater than this. In the above calculation we have used a simple model for the toroidal geometry and free energy. Several objections could be raised against this model. We raise some of these here and then refine our calculation to take them into account. The first is that, as shown by Ubbink and Odijk [15], the cross-sectional shape is not necessarily circular. In fact as $\alpha$ becomes large the shape can 
distort so it is flattened in the center. This only becomes significant for large values of $\alpha$ of order 3, and our transition occurs for $\alpha \approx 0.8$. Nevertheless, it is something that should be accounted for, if only as a precaution. Second, it is rather clear that one advantage the sphere has over the simple toroid is that in a sphere the winding is around a point (the center) whereas in the toroid the winding is assumed to be about a central axis. This, however, need not be the case. In fact chains could wind about the central point of the toroid and hence reduce their bending energy. In the following we account for both these effects. To account for the distortion in shape of the toroid we again use a plane polar coordinate system, but with the dimensionless radius $\rho$ being a function of $\theta$. We let $\mathcal{R}_{c}$ be the distance of the centroid of our shape from the toroidal center. We use the same dimensionless energies and lengths as above. The bending free energy is then

$$
\mathcal{F}_{\text {bend }}=\pi \int_{0}^{2 \pi} d \theta \int_{0}^{\rho(\theta)} d r r\left(\mathcal{R}_{c}^{2}+r^{2}+2 \mathcal{R}_{c} r \cos \theta\right)^{-1 / 2} .
$$

The integral over $r$ can be done explicitly leaving only a functional of $\rho(\theta)$.

The surface energy can be obtained using the theorem of Pappus:

$$
\mathcal{F}_{\text {sur } f}=2 \pi \alpha \mathcal{R}_{c} \int_{0}^{2 \pi} d \theta \sqrt{\rho^{2}+(d \rho / d \theta)^{2}} .
$$

The major radius can be eliminated by calculating the volume using another theorem of Pappus:

$$
1=\pi \mathcal{R}_{c} \int_{0}^{2 \pi} d \theta \rho^{2}(\theta) .
$$

We need to minimize $\mathcal{F}_{\text {bend }}+\mathcal{F}_{\text {sur } f}$ subject to the constraints that the origin of the $(r, \theta)$ coordinate system lies at the centroid:

$$
\int_{0}^{2 \pi} d \theta f(\theta) \rho^{3}(\theta)=0
$$

where $f(\theta)=\sin (\theta)$ and $\cos (\theta)$.

We can now minimize our free energy as a function of $\alpha$ and obtain a modified form of the free energy for a torus. However, this is very close to that of our previous simple model for $\alpha<1$. This means that there is negligible change to the critical value of $\alpha$, which remains at $\alpha_{c} \approx 0.8423$.

At the critical value of $\alpha$ the cross section of the toroid is very close to being circular. This means that the distorted cross sections predicted by Ubbink and Odijk for large $\alpha$ toroids will never exist in equilibrium, since for these large values of $\alpha$ we find a hollow sphere is more stable. However, our work does not entirely rule out such distorted toroids, as they might exist as a local equilibrium morphology, even if the global equilibrium is not a torus. It is important to compare our work with some of the previous work relating to toroids. In particular, Park et al. [12] have suggested that packing defects can play an important role in determining toroidal size for the low-density case where a virial expan- sion in the polymer density is appropriate. It is somewhat difficult to compare our work with theirs, since the work of Park et al. concerns dilute toroids whereas ours work concerns a "melt toroid." Furthermore, Park et al. do not actually present any graphs of toroidal radius versus $\alpha$. In a melt we might expect that packing defects would be more important. However, even in a melt, a lot of the space is actually empty, so that it is fair to say that the defects suggested by Park et al. can be avoided. Thus, the chains can avoid sharp bends while crossing each other, because a significant amount of empty space is provided to them. What we have shown here is that such defects are not needed to explain the small variation of radii in toroids.

We should also remark on some recent work by Vasilevskaya et al. [17], who have suggested that spherical globules can exist, in the low-density limit. This is based on the observation that the toroids get fatter as the amount of material is increased, and eventually the major and minor radii become equal. What we have shown is that this is not an accurate way of estimating the crossover point, since in fact a hollow sphere becomes favored when the toroid is still very thin. In Fig. 5 of Vasilevskaya et al. [17] evidence is provided for a "quasi-spherical" geometry for the T4-DNA molecule. However, whether the structure has a hole in the middle is impossible to determine. It must be also noted that both Monte Carlo and Brownian dynamic simulations do observe a transition from a planar, toroidal structure to a spherical globule structure on increased chain length, at fixed bending constant $[10,19,20]$. However, it is not clear from the simulations that a hole forms at the center of the spherical structure. Indeed, given that we have shown above that this hole is quite small, it could be quite difficult to clearly identify its presence from coarse-grained simulations.

In this article we do not consider twist energy. Indeed, we assume our polymers have a negligible twist constant. For some double-stranded biopolymers this may not be a valid assumption [21]. In this case we anticipate the twist energy would have a much greater effect on the spherical globule than on the (planarlike) toroidal geometry. (In the toroidal geometry for most of the chain length the chain can be considered in one planar layer.) Thus we would expect the transition from toroidal to hollow sphere would occur at a larger $\alpha$ value.

\section{MANY-CHAIN SYSTEMS AND DIFFERENT MORPHOLOGIES}

We now consider the scenario where we have many chains in our system. The first morphology we consider, as for a single chained system, is a thickened toroid. The free energy for many chains is calculated as before, except we have the volume constraint that the volume of the toroid is $\mathcal{N} L a^{2}$, where $\mathcal{N}$ is the number of chains in the system. The free energy of the thickened toroidal condensate may then be easily evaluated as a function of $\mathcal{N}$.

The next possible configuration of the semiflexible polymer condensate we consider is a spherical globule. To determine the free energy for this configuration is a complicated task, not least because it is not straightforward as to how the 
stiff tubes will pack into a sphere. For the single-chained system we neglected any packing problems for the spherical geometry. Here, we must address this question because it is apparent the spherical geometry will not have have a hollow center. For example, close to the center of the sphere, because there are many chains present, the chains could pack radially and so be effectively rigid. This would only occur close to the center of the sphere, further out the chains would begin to wrap into the spherical geometry. Thus the problem of large (possibly infinite) bending at the center of the sphere is avoided. To be able to calculate the free energy for a spherical geometry we shall adopt a simplified packing structure which is not necessarily the optimal packing structure for the tubes. In fact, it most likely is not; however, our structure will give an estimate of the free energy for a spherical globule. This estimate will be useful in deciding (approximately) when such a structure will become a stable state.

We assume the globule is made up of a series of discs. Within each disk the chains initially pack radially and then at some radius $R_{0}$ they bend and pack in concentric circles; see Fig. 4(a). We need to initially determine the trajectory of a "typical" chain, which in turn will allow us to determine its bending energy. First we determine the number of chains which will fully pack a circular disk to radius $R_{0}$. To do this we use a similar method to one given by Williams and Fredrickson [22] for the packing of rods in a sphere. We divide two-dimensional (2D) space into circular shells of thickness $l$ and divide the $j$ th shell at radius $j l$ into $n_{j}$ shells of volume $l a^{2}$ so that $n_{j}=2 \pi j(l / a)$. If we let $m_{j}$ be the number of tubes beginning in the $j$ th layer, then the number of available sites for starting tubes in layer $i$ is $\bar{n}_{i}$ and given by $\bar{n}_{i}=2 \pi i(l / a)$ $-\sum_{j=1}^{i-1} m_{j}$. In the continuum limit where the radius of the sphere, $R_{0}$, is much larger than $l$, we have $i=R_{0} / l$ and the number of rods starting between $R_{0}$ and $R_{0}+d R_{0}$ is $m\left(R_{0}\right) d R_{0}$. Assuming that $R_{0}<L$, which is most likely for long chains, then the number of available sites for new chains at the $i$ th layer is $\bar{n}_{i}-2 \pi\left(R_{0} / a\right)-\int_{0}^{R_{0} / a} m(r) d(r / a)$. Now in the continuum limit one can show $\bar{n}_{i}=\operatorname{am}\left(R_{0}\right)$ so that we get the following first-order differential equation for $m\left(R_{0}\right)$ :

$$
\frac{d m\left(R_{0}\right)}{d R_{0}}=\frac{2 \pi}{a}-\frac{m\left(R_{0}\right)}{a} .
$$

The solution is straightforward and is $m\left(R_{0}\right)=2 \pi[1$ $\left.-\exp \left(-R_{0} / a\right)\right]$. Thus the total number of tubes that pack a circular disk up to radius $R_{0}$ is $N\left(R_{0}\right)$ and is $N\left(R_{0}\right)=\Sigma m_{j}$ $=\int_{0}^{R_{0} / a} m(r) d(r / a)$. On completing the integral we find $N\left(R_{0}\right)=2 \pi\left(R_{0} / a\right)\left[1-\left(a / R_{0}\right)+\left(a / R_{0}\right) \exp \left(-R_{0} / a\right)\right]$. Now the radius of the disk, $R_{0}$, would be expected to be much larger than $a$ (certainly for many chains, where we would expect the spheres to become stable) so that $a / R_{0} \ll 1$ and to leading order $N\left(R_{0}\right)=2 \pi\left(R_{0} / a\right)$. Essentially, the number of chains emerging from the circular disk is equal to the perimeter area of the disk divided by the cross-sectional area of the tubes.

If the tubes pack radially (initially) to some radius $R_{0}$ and if all these chains formed a disk of thickness $a$ units, the total radius $R_{\text {out }}$ of the disk is given by $\pi R_{\text {out }}^{2} a=N L a^{2}$, so that

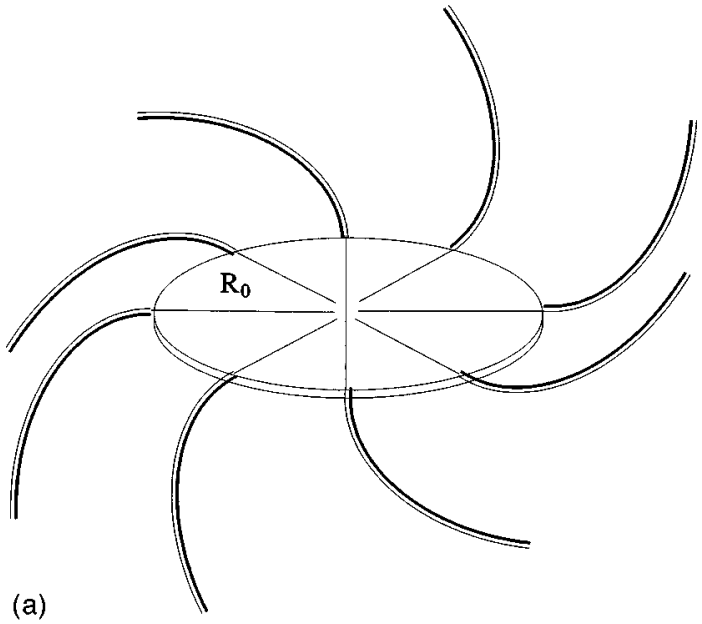

(b)

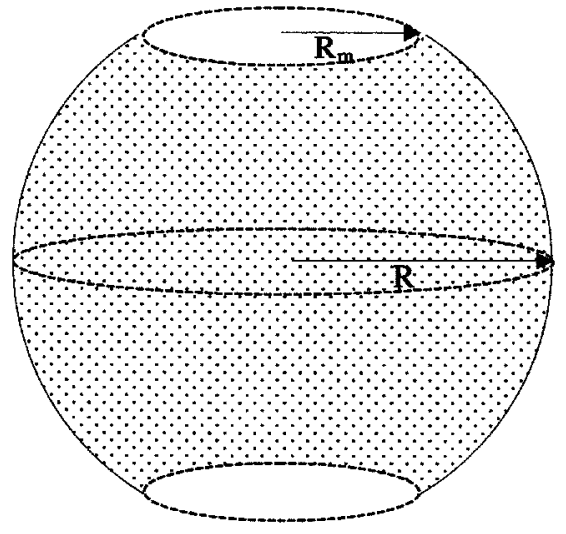

FIG. 4. (a) Schematic of a typical disc of semi-flexible chains. (Note the figure should be viewed as if all chains and disk are in the same plane. Not all chains in disk are drawn.) The chains pack the disc up to radius $R_{0}$ and then begin to wrap around each other in the same disk. (b) A schematic of the pseudospherical globule. The ends are chopped off so that the final disk (top and bottom) has $a_{0}$ chains and corresponding radius $R_{m}$. The radius of the sphere is $R$.

$R_{\text {out }}=\left(2 L R_{0}\right)^{1 / 2}$. Note that if $l$ is the length a chain expends, on average, to get to the cylindrical surface of radius $R_{0}$ then $\pi R_{0}^{2} a=N l a^{2}$, which gives $l=R_{0} / 2$. At $r=R_{0}$ the tubes emerge at right angles to the tangent to the circle. [As a simplification one may understand this in the following way: Consider a cylinder of radius $R_{0}$ and height $a$ and to this cylinder are grafted $N\left(R_{0}\right)$ semiflexible chains. One now asks, what is the bending energy associated with this semiflexible polymer brush, as they wrap round the surface of the cylinder?] At this point they begin to bend away from their radial trajectory into concentric circles (or spirals). At each incremental length $\delta r$, the number of chains that cross the radius $R_{0}+\delta r$ must be still $N$. However, the extra length available to the chains must be taken up with the decreased tilt angle (to the radial tangent). The tilt angle initially is $\pi / 2$ and this decreases as we begin to wind. The length each chain takes up on the circle at radius $R_{0}+\delta r$ is $a / \sin \phi$ where $\phi$ is the tilt angle. As $r \rightarrow \infty$ we see the tilt angle $\phi$ goes to zero (as expected). So at any radius $R_{0}+\delta r$ we have the equality $\mathrm{Na} / \sin \phi=2 \pi\left(R_{0}+\delta r\right)$ and substituting for $N$ we find at any radius $r, r=R_{0} / \sin \phi$. Now we require $\phi$, the tilt angle at any 
radius, in terms of $r$ and $\theta$, the polar angle. If $\hat{\mathbf{n}}$ is a unit vector along the chain trajectory and $\hat{\mathbf{m}}$ is a unit vector along the tangent to the circle of radius $r$, then $\cos \phi=\hat{\mathbf{n}} \cdot \hat{\mathbf{m}}$. In polar coordinates the unit tangent vector to the circle is $\hat{\mathbf{e}}_{\theta}$ and a unit vector along the chain is $\hat{\mathbf{s}}=\left(d r \hat{\mathbf{e}}_{r}+r d \theta \hat{\mathbf{e}}_{\theta}\right) /\left[r^{2}\right.$ $\left.+r_{\theta}^{2}\right]^{1 / 2}$. Thus $\cos \phi=r(\theta) /\left[r^{2}+r_{\theta}^{2}\right]^{1 / 2}$ and so $\sin \phi=r_{\theta} /\left[r^{2}\right.$ $\left.+r_{\theta}^{2}\right]^{1 / 2}$. For simplicity we denote $d r / d \theta \equiv r_{\theta}$. (Alternatively, we know $\tan \psi=r d \theta / d r$ where $\psi$ is the angle between the tangent to the curve and the radial vector. So $\psi=\pi / 2-\phi$. From this one can show, once again, $\sin \phi=r_{\theta} /\left[r^{2}+r_{\theta}^{2}\right]^{1 / 2}$.) The desired relationship between tilt angle and polar angle thus yields the ordinary differential equation $r=R_{0}\left(r^{2}\right.$ $\left.+r_{\theta}^{2}\right)^{1 / 2} / r_{\theta}$. This equation may be solved analytically with the solution

$$
\theta=\theta_{0}+\left\{\left[\left(\frac{r}{R_{0}}\right)^{2}-1\right]^{1 / 2}-\arctan \left[\left(\frac{r}{R_{0}}\right)^{2}-1\right]^{1 / 2}\right\},
$$

where $\theta_{0}$ is the initial polar angle of a particular chain. Each chain in the disk (of initial radius $R_{0}$ ) has the same trajectory, given by Eq. (15). Note that for large $r\left(r \gg R_{0}\right)$ the trajectory becomes $\theta-\theta_{0}+\pi / 2=r / R_{0}$ or $r=R_{0} \Delta \theta$, where $\Delta \theta$ is the (effective) polar angle. Given that $R_{0}=N a /(2 \pi)$ we see that an increase in polar angle of $2 \pi$ corresponds to the trajectory increasing by a radius of $\mathrm{Na}$, as expected for wrapping of chains. Furthermore, the arc length of such a chain is given by $s=\int_{R_{0}}^{R_{\text {out }}} d r\left[1+\left(r \theta_{r}\right)^{2}\right]^{1 / 2}$. This integral may be easily completed with Eq. (15) to yield $s=L-R_{0} / 2$, which is as expected.

Now we require the bending energy which is given by

$$
\frac{F_{\text {bend }}}{k_{B} T}=\frac{P}{2} \int_{R_{0} / 2}^{L} c^{2}(s) d s=\frac{P}{2} \int_{r_{\text {inn }}\left(R_{0}\right)}^{r_{\text {out }}(L)} c^{2}(r)\left[1+\left(r \theta_{r}\right)^{2}\right]^{1 / 2} d r .
$$

Here $s$ is the arc length along the polymer chain. We need $c(r)$ for the bending energy which is calculated as $c(r)$ $=\left(r^{2} \theta_{r}^{3}+2 \theta_{r}+r \theta_{r r}\right) /\left(1+r^{2} \theta_{r}^{2}\right)^{3 / 2}$. In fact this turns out to be relatively simple for our trajectory (15)-i.e., $c$ $=R_{0}^{-1}\left[\left(r / R_{0}\right)^{2}-1\right]^{-1 / 2}$ which we may use to calculate the bending energy. Thus the bending energy integral becomes

$$
\frac{F_{\text {bend }}}{k_{B} T}=\frac{P}{4 R_{0}} \int_{1}^{\rho_{\text {outer }}} \frac{\rho}{\rho^{2}-1} d \rho,
$$

where $\rho_{\text {outer }}=2(\pi L / a)^{1 / 2} / N^{1 / 2}$. The integral may be readily completed and involves the natural logarithm of the denominator. When this is evaluated at the bottom terminal, it yields a weak (logarithmic) divergence. Physically, the reason for this divergence is the following. Our packing constraint is initially (i.e., at $r=R_{0}$ ) too strong and forces the chain to bend too sharply. In reality, there is no need to force such a strict restriction on the chains, as there is sufficient free volume present for the chains to overcome such a sharp bend. Mathematically, to overcome the weak divergence we shall calculate the bending energy from one monomer away from the initial point $r=R_{0}$. Thus rather than having a lower ter- minal of 1 in Eq. (17) we have a lower terminal of $\left(R_{0}\right.$ $+a) / R_{0}=1+\left(a / R_{0}\right)$.

The globule is made up of stacks of disks and in each disk layer the chains wind as discussed above. Initially we shall consider the globule to be made up of disks but in each layer there is one less chain. This results in a globule shape which has the approximate shape of a cone of stacked disks (the cone is not smooth but has a steplike boundary shape). One extra problem is that we cannot let the number of chains at either of the ends go to 1 , because if it did, the continuum approximation breaks down. In addition, this could lead to an infinite curvature and hence divergent free energy. Hence, we impose a lower cutoff of $a_{0}$ chains in each of the end layers. We can calculate the surface energy quite readily for such a geometry and the bending energy using the calculation above. The free energy for this geometry, however, is not less than the energy of a thick toroid for any $\mathcal{N}$. It is apparent from this calculation that the major contribution to the globules free energy is its surface energy. For the cone of stacked disks shape the major reason the free energy is large is that, because each layer has one less chain, the additional surface area increases in discrete jumps. It is now clear if we are to obtain a spherical shaped globule we need to let chains fill the gaps between layers-i.e., smooth out the steplike increases.

To do this we implement the following model. The globule has $\mathcal{N}$ chains in total. If it were to form a perfectly spherical shape, then the radius of the globule, $R$, would be related to the number of chains via $4 \pi R^{3} / 3=\mathcal{N} L a^{2}$. However, we shall still consider our globule to be made up of disk of chains. In the continuum limit, where our model is valid, we have a lower cutoff on the number of chains in the end layers. This cutoff is $a_{0}$ chains. (We can vary $a_{0}$, but we shall see the results are independent of this artifact.) Thus the minimum radius of a layer (i.e., layers at top and bottom extremities), $R_{m}$, is given by $\pi R_{m}^{2} a=a_{0} L a^{2}$. The globule thus is a sphere but its ends are chopped off; see Fig. 4(b). Thus the volume of the globule is $4 \pi R^{3}\left[1-\left(R_{m} / R\right)^{2}\right]^{1 / 2}[1$ $\left.+\left(R / R_{m}\right)^{2} / 2\right] / 3$, and since the globule is assumed to be incompressible and contains no solvent, we have a relationship which may be used to determine $R$ :

$$
\mathcal{N} L a^{2}=\frac{4}{3} \pi R^{3}\left[1-\left(R_{m} / R\right)^{2}\right]^{1 / 2}\left[1+\left(R / R_{m}\right)^{2} / 2\right] .
$$

In the limit of $R_{m} \ll R$, which we believe is the case for many chains, the square root in the above equation can be expanded to the second term. The resultant equation turns out to be a quartic equation for $R$ which, fortunately, has only one physically correct root which is given by

$$
R=\frac{u_{1}^{1 / 2}}{2}\left\{1+\left[2\left(1+\frac{R_{m}^{4}}{u_{1}^{2}}\right)^{1 / 2}-1\right]^{1 / 2}\right\},
$$

where $\quad u_{1}=v_{1}+v_{2}, \quad v_{1}=\left(q+\Delta^{1 / 2}\right)^{1 / 3}, \quad v_{2}=\left(q-\Delta^{1 / 2}\right)^{1 / 3}, \quad q$ $=9 V^{2} /\left(32 \pi^{2}\right), \Delta=R_{m}^{12} / 27+(3 V / 4 \pi)^{4} / 4$, and $V=\mathcal{N} L a^{2}$. Now that we have the radius we can calculate the surface energy of the globule, which is the surface tension times the surface area. Thus $F_{\text {sur } f}=2 \pi R^{2} \gamma\left\{2\left[1-\left(R_{m} / R\right)^{2}\right]^{1 / 2}+\left(R_{m} / R\right)^{2}\right\}$. Consider now a layer which is ia (where $i=1,2,3, \ldots$ ) units 

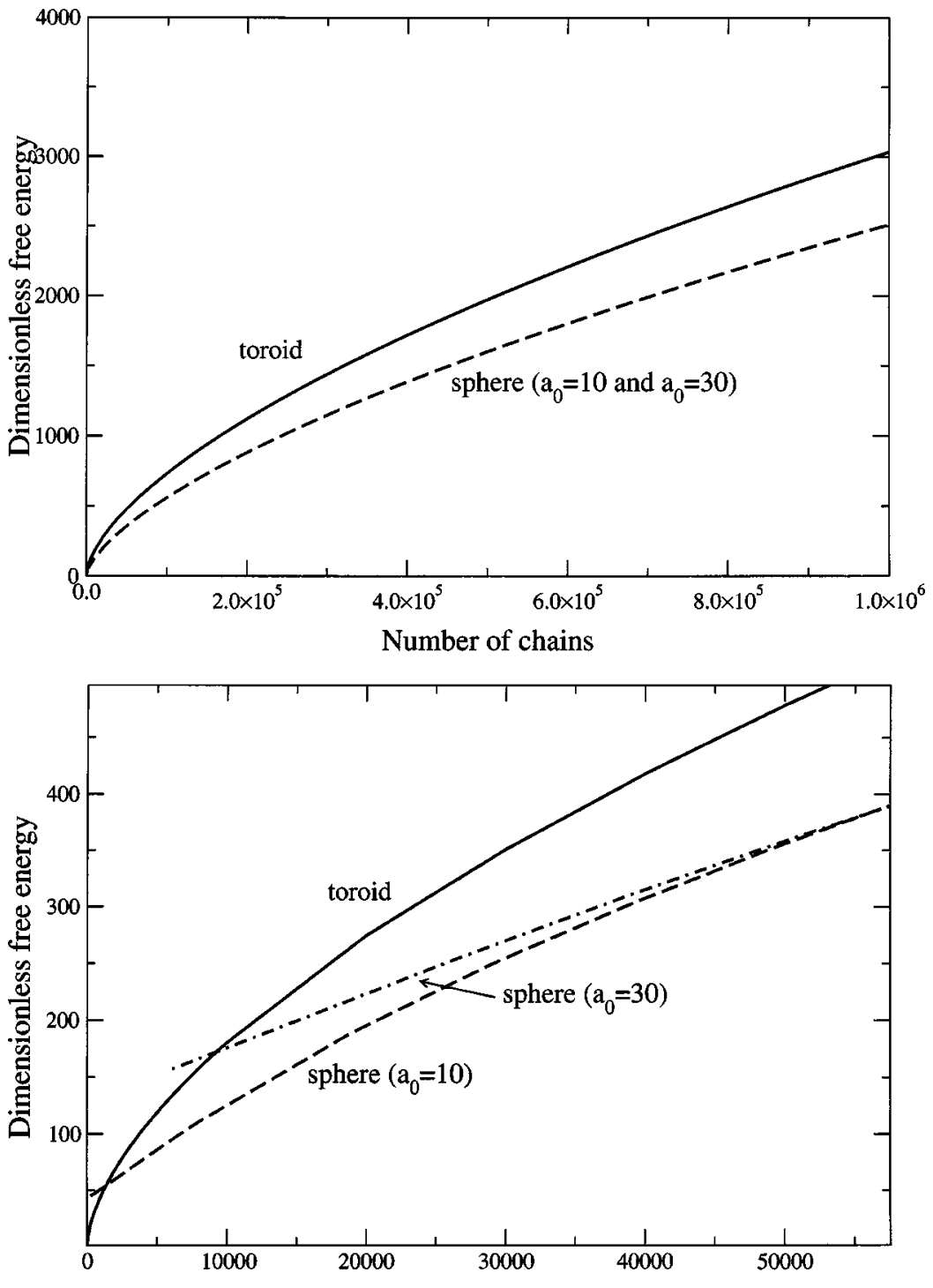

(b)

Number of chains

FIG. 5. Dimensionless free energy $\mathcal{F}$ of a spherical globule (dashed line $-a_{0}=10$, dot-dashed line $-a_{0}=30$ ) and thick toroid (solid line) versus number of chains, $\mathcal{N}$. (a) Up to $10^{6}$ chains (the $x$ axis is in units of $10^{5}$ chains). (b) For fewer chains showing the crossover from toroids to spheres.

above the middle layer. Its radius would then be $R(i)=\left[R^{2}\right.$ $\left.-(i a)^{2}\right]^{1 / 2}$. The maximum number of chains that could reside in this layer (without carrying outside the boundary, $R(i)$,) is given by the integer $N(i)$ between $\pi\left(R^{2}(i) / L a^{2}\right)-1<N(i)$ $<\pi\left(R^{2}(i) / L a^{2}\right)$. The additional space that at the moment remains void in each layer is therefore $\pi R^{2}(i) a-\pi \rho^{2}(i) a$ where $\pi \rho^{2}(i) a=N(i) L a^{2}$. This volume may be filled up with additional chains which wrap around in spirals (and whose bending energy is easily evaluated). However, to exactly fill each layer out to the required radius - i.e., $R(i)$ - these additional chains must traverse into adjacent layers. To do this the perfect packing arrangement of the stacked cone geometry is not maintained close to the surface of the sphere. We neglect the small inaccuracy which this involves; e.g., the free volume is not accounted for correctly and there may be an additional bending and twisting energy involved in chains traversing from layer to layer. We can now calculate the total free en- ergy of a globule numerically, for incremental total number of chains.

The results of our calculation are shown in Fig. 5, where we plot dimensionless free energy $\mathcal{F} \equiv F a^{2} \epsilon^{-1} V^{-1 / 3}$ versus $\mathcal{N}$ - the number of chains in the globule. We use values of $L$, $a, \gamma$, and $P$ such that $\alpha=10^{-4 / 3} \approx 0.046$ so that a single chain would form a toroid, according to Sec. II. (For this semiflexible chain the region of stability for toroids is $3 \times 10^{-7} \leqslant \alpha$ $\leqslant 0.8423$.) In Fig. 5(a) we show the free energy up to $10^{6}$ chains and it is clear that the spherical globule energy is much less than the thickened toroidal free energy, indicating stability for the sphere. The actual crossover from a thickened toroid to sphere happens to occur at much lower $\mathcal{N}$. Figure 5(b) shows the free energy at lower $\mathcal{N}$. Here we show results for two different values of $a_{0}$. (Recall that $a_{0}$ is the number of chains in the end layers and is an artifact which we introduced to simplify our model.) We see for both values of $a_{0}$ that there exists a crossover from toroid to sphere, the 
larger the $a_{0}$ the larger the crossover, as one would expect. In fact for $a_{0}=10$ the crossover is at approximately $\mathcal{N}=1500$ and for $a_{0}=30$ the crossover is at roughly $\mathcal{N}=9500$. Both these values are large enough, not to violate our continuum approach. Note that for large $\mathcal{N}$, these two curves merge together, indicating that the effect of introducing the artifact $a_{0}$ becomes negligible as the number of chains in the system becomes large. Hence our results are qualitatively independent of the value of $a_{0}$. Our calculations have shown that at low chain concentration a toroidal geometry should be stable while at sufficiently high chain concentrations the spherical geometry should be stable. A first-order phase transition (from toroids to spheres) is predicted at the critical chain concentration where the crossover occurs. Recently Conwell et al. [23] have prepared toroidal DNA condensates from salt solutions. The toroidal DNA condensate has a mean toroid diameter, $2 R$, of $55 \mathrm{~nm}$ and a mean toroidal (cross-sectional) thickness, $2 r$, of $25 \mathrm{~nm}$. From this they estimate the condensate has 6-32 DNA chains. It is hard to compare our theory with Conwell et al. since we do not know their effective $\alpha$ value. However, an estimate of around 1000 chains for the transition, from our theory, does not seem unreasonably large, given that this is an upper estimate.

Finally, we emphasize that our calculations only give upper estimates of the free energy for the spherical globule. Since we have implemented a model where the chains essentially wrap in parallel disks, we have had to introduce the simplification that the sphere has its ends chopped off (i.e., the minimum chains in end layers is $a_{0}$ ). Of course real spherical globules will not have such constraints and thus their energies will be smaller (per chain) compared to our energies. It is also most probable that there will be a better packing arrangement, in general, than what we have used which would lead to a lower free energy. In fact, very recently Kulic et al. [24] have analyzed a novel packing arrangement of the tubes in a toroidal condensate. In their model [24] they consider the tubes to run at some angle to the main toroidal plane. They find such a packing arrangement may be the real arrangement for thicker toroids. Indeed, it is possible such an arrangement might also be useful for the dense spherical globule. Our spherical energy is therefore just an upper estimate of a real spherical globule's free energy. From this estimate we can conclude that at sufficiently high chain numbers (or concentrations) the condensed globule will be spherical rather than a toroid. The crossover number of chains we have predicted from our models are just rough estimates. In reality we would expect much lower crossovers than predicted here (since our spherical globule energies are upper bounds). We hope this work will stimulate further more accurate numerical models and experiments on many chained systems to observe such a crossover.

\section{SUMMARY}

In this article we have considered the possible morphologies formed from (stiff) semiflexible polymers when they are put in a poor solvent (or with a condensing agent). We have initially studied the the effect of persistence length versus overall length of a single polymer chain. We have shown above a critical value of the dimensionless parameter $\alpha$ $\equiv a^{2} V^{1 / 3} \gamma \epsilon$ a single semiflexible chain will form a hollow sphere. This value is $\alpha_{c} \approx 0.84$. Thus on increasing $\alpha$ we find initially rods, then toroids and finally spheres. We have also considered the case of many semiflexible chains in a poor solvent. An individual chain will form a toroid, but the question is whether many such chains will also form a toroid? We have shown there exists a critical number of chains (or concentration) above which the chains will form a densely packed sphere. Below this concentration the chains will condense to a thickened toroid,

There are obvious applications of the hollow sphere morphology discussed here. These are all applications in which surfactant vesicles are currently involved, most notably in the cosmetic and "controlled-release" drug industries. The semiflexible polymer system has certain differences with surfactant vesicles which might be useful. Most notably, the walls are thick, thus leading to slower release, and can be of controlled thickness, by adjusting the chain length. In practice some precautions would need to be taken to ensure that the chains did not decondense. This could be done for instance by crosslinking after condensation.

To conclude this article we turn from hard calculation to an observation. In all the forms of life with which the authors are familiar the genetic material (DNA or RNA) is encapsulated in some shell of lipids or proteins. Our hollow sphere configuration allows for a novel form of encapsulation where the genetic material itself is the shell.

\section{ACKNOWLEDGMENT}

G.G.P. acknowledges financial support from ARC QEII.
[1] P. G. de Gennes, Scaling Concepts in Polymer Physics (Cornell University Press, Ithaca, NY, 1979).

[2] V. A. Bloomfield, Curr. Opin. Struct. Biol. 6, 334 (1996).

[3] Yu. M. Evdokimov, T. L. Pyatigorskaya, O. F. Polyvtsev, N. M. Akimenko, V. A. Kadykov, D. Ya. Tsvankin, and Ya. M. Varshavsky, Nucleic Acids Res. 3, 2353 (1976).

[4] N. V. Hud, Biophys. J. 69, 1355 (1995).

[5] N. V. Hud, K. H. Downing, and R. Balhorn, Proc. Natl. Acad. Sci. U.S.A. 92, 3581 (1995).
[6] Y. Fang and J. H. Hoh, Nucleic Acids Res. 26, 588 (1998).

[7] U. K. Laemmli, Proc. Natl. Acad. Sci. U.S.A. 72, 4288 (1975).

[8] L. S. Lerman, Proc. Natl. Acad. Sci. U.S.A. 68, 1886 (1971).

[9] K. Minawa, Y. Matsuzawa, K. Yoshikawa, M. Doi, and A. R. Khokhlov, Biopolymers 34, 555 (1994).

[10] H. Noguchi and K. Yoshikawa, J. Chem. Phys. 109, 5070 (1998).

[11] J. Ubbink and T. Odijk, Biophys. J. 68, 54 (1995). 
[12] S. Y. Park, D. Harries, and W. M. Gelbart, Biophys. J. 75, 714 (1998).

[13] G. G. Pereira and D. R. M. Williams, Biophys. J. 80, 161 (2001).

[14] V. V. Vasilevskaya, A. R. Khokhlov, Y. Matsuzawa, and K. Yoshikawa, J. Chem. Phys. 102, 6595 (1995).

[15] J. Ubbink and T. Odijk, Europhys. Lett. 33, 353 (1996).

[16] J. N. Bright and D. R. M. Williams, Europhys. Lett. 45, 321 (1999).

[17] V. V. Vasilevskaya, A. R. Khokhlov, S. Kidoaki, and K. Yoshikawa, Biopolymers 41, 51 (1997).

[18] G. G. Pereira and D. R. M. Williams, Europhys. Lett. 50, 559 (2000).
[19] V. A. Ivanov, M. R. Stukan, V. V. Vasilevskaya, W. Paul, and K. Binder, Macromol. Theory Simul. 9, 488 (2000).

[20] I. R. Cooke and D. R. M. Williams, Physica A 339, 45 (2004).

[21] R. Golestanian and T. B. Liverpool, Phys. Rev. E 62, 5488 (2000).

[22] D. R. M. Williams and G. H. Fredrickson, Macromolecules 25, 3561 (1992).

[23] C. C. Conwell, I. D. Vilfan, and N. V. Hud, Proc. Natl. Acad. Sci. U.S.A. 100, 9296 (2003).

[24] I. M. Kulic, D. Andrienko, and M. Deserno, Europhys. Lett. 67, 418 (2004). 